\title{
Prototyping Strategies for the Agile Development of Additive Manufactured Products: A Case Study from the COVID-19 Pandemic
}

\author{
Daniel Omidvarkarjan ${ }^{1 *}$, Ralph Rosenbauer ${ }^{2}$, Daniel Kirschenbaum ${ }^{3}$, Daniele \\ Cipriano ${ }^{2}$, David Ochsner ${ }^{2}$, Harry Woodfin ${ }^{2}$, Christoph Klahn ${ }^{1}$, Mirko Meboldt ${ }^{4}$ \\ ${ }^{1}$ Inspire AG \\ 2 HelpfulETH, ETH Zürich \\ ${ }^{3}$ Institut für Neuropathologie, Universitätsspital Zürich \\ ${ }^{4}$ Product Development Group Zurich (pd|z), ETH Zürich \\ * Corresponding author: \\ Daniel Omidvarkarjan \\ Inspire AG \\ Leonhardstrasse 21 \\ 8092 Zürich, Schweiz \\ Telefon: + 41 (0)446327119 \\ Mail: omidvarkarjan@inspire.ethz.ch
}

\begin{abstract}
Frequent validations of physical product increments play an essential role for Agile Hardware Development (AHD). This paper presents practices for the accelerated embodiment of increments based on the analysis of an AHD project. It describes adaptations of established prototyping strategies for the use of Additive Manufacturing (AM), covering iterative refinement, parallel development, prototyping media change and scheduling of build phases. The application of the practices led to an accelerated embodiment process with mean iteration lengths of 6 days and a first release of a viable product increment within 18 days. By enabling early and frequent validations with tangible increments, the practices facilitate the effective application of AHD in practice.
\end{abstract}

\section{Keywords}

Agile hardware development, prototyping strategies, design for additive manufacturing 


\section{Introduction}

Agile Hardware Development (AHD) has proven to be a viable development approach for the rapid creation of physical systems [1]. It is especially suited for projects with high uncertainty regarding user requirements and technical implementation. Compared to traditional NPD approaches, the application of Agile offers several benefits including shorter time-to-market, less risk and higher responsiveness to change [2].

For physical products, several hardware specific hurdles hinder the application of AHD. These are summarized as constraints of physicality (CoP) and include for instance the timeconsuming \& costly build process of increments [3]. These constraints create a major implementation challenge as AHD necesarily relies on the frequent design, build and test of physical increments. They serve to reduce uncertainty and provide a productive output for users [4]. The use of rapid prototyping technologies such as Additive Manufacturing (AM) can lessen the impact of CoP, as it enables the economical production of small lot sizes [5]. Nevertheless, in practice the challenges of CoP are still present for more sophisticated AM processes such as Selective Laser Sintering (SLS) or Selective Laser Melting (SLM). Due to their significantly higher cost structure (e.g. compared to Fused Deposition Modeling) and longer processing times (e.g due to cool down phases) [6], frequent iterations become timeconsuming \& costly.

To support the prototyping process, studies like [7] have aggregated prototyping strategies on a generic level. Within their contribution, the authors of [8] present various strategic factors that designers can leverage to plan the embodiment of prototypes. These include prototyping media (physical vs. virtual), scale (reduced vs. final), strictness of requirements (relaxed vs. final) and system level (isolated vs. integrated). Additional techniques in their paper encompass iterative or parallel prototyping. The descriptions are on a generic level and therefore not address the context of AHD and the constraints of a respective manufacturing technology such as AM. To sum up, little guidance is provided on how the development of AM increments within an AHD project can be facilitated to enable an effective application of AHD.

\section{Objective and Method}

The goal of this study is therefore to answer the following research question: How can the CoP of $A H D$ be addressed with AM-specific prototyping strategies to facilitate the embodiment of physical increments?

To answer the research question, an exemplary AHD project (illustrated in Figure 1) of the COVID-19 pandemic is retrospectively analyzed (see Figure 2). The subject of the case study is the PACID system (Pathogen Containment for Interactive Devices). The solution aims to shield portable electronic devices (such as phones or pagers) from germs through a transparent bag.

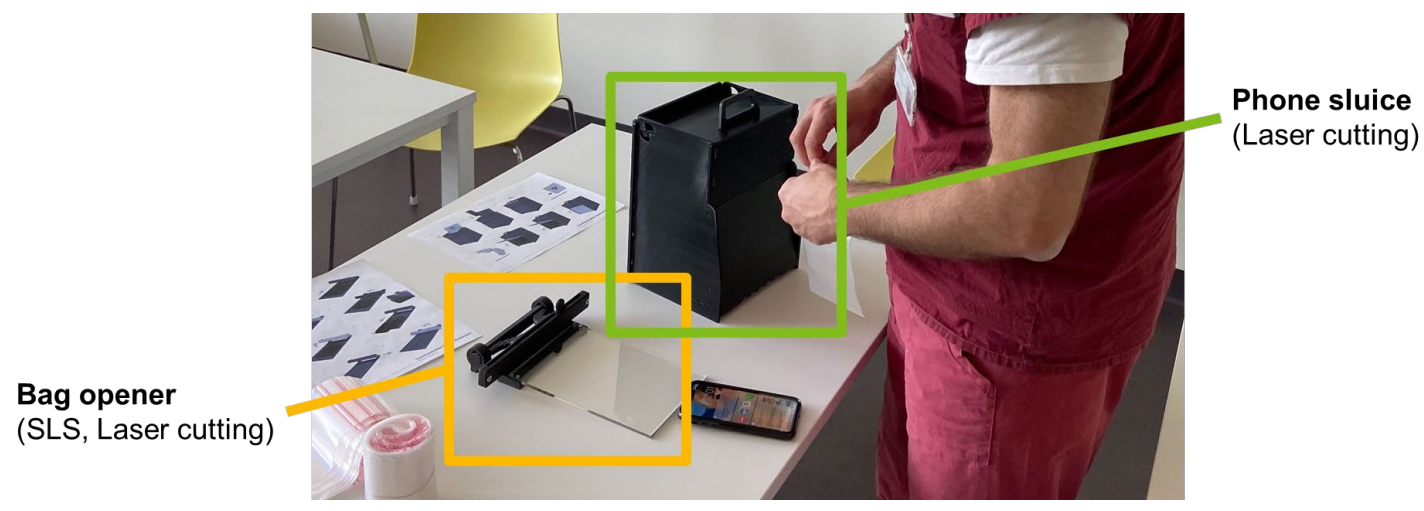

Figure 1: PACID system with its components (bag opener \& phone sluice) 


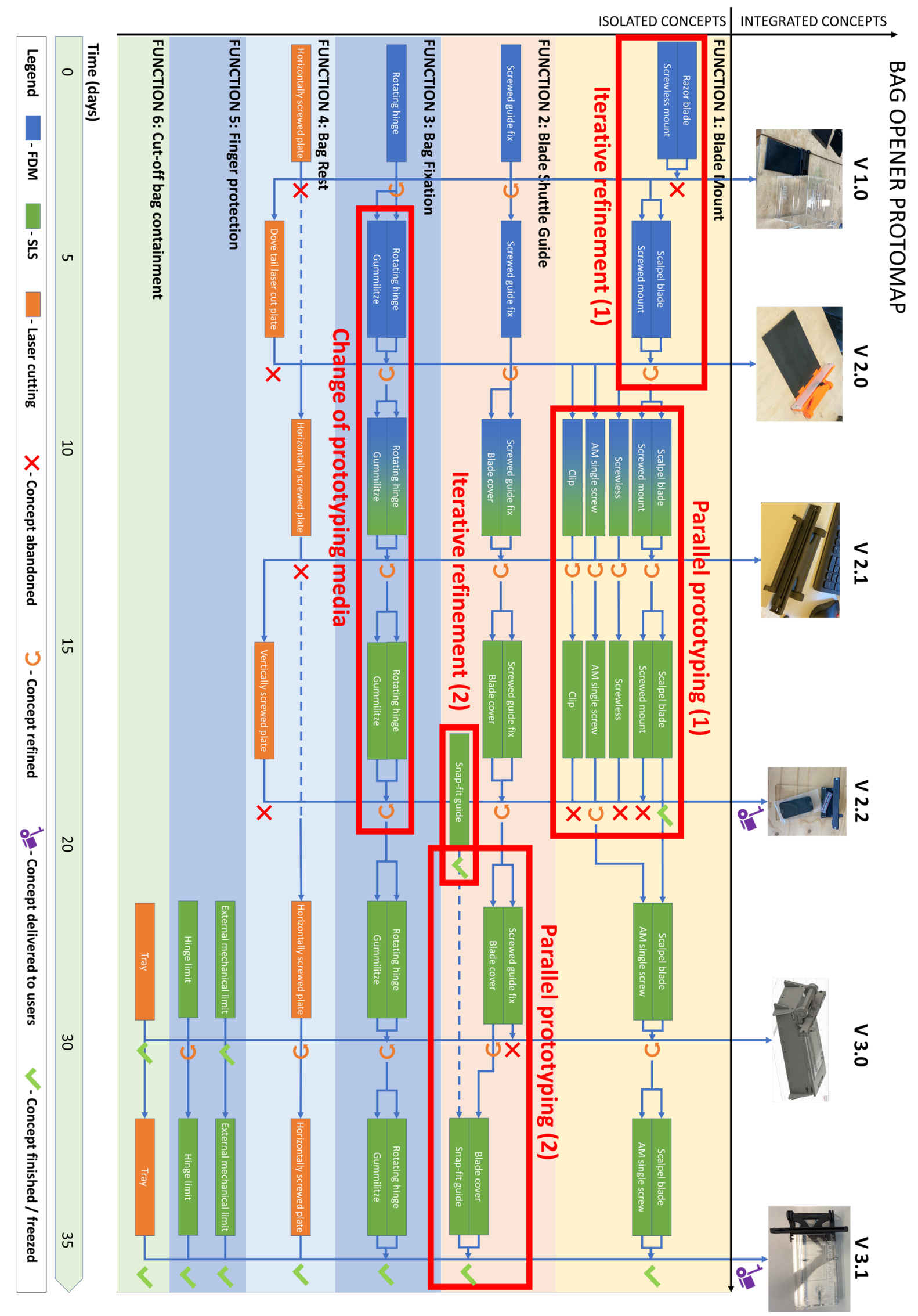

Figure 2: Protomap of the bag opener assembly with identified prototyping strategies in red 
Healthcare professionals can maintain access to the phone's features without risking surface contamination. The PACID system consists of a SLS bag opener and a laser cut sluice that stores the electronic device until it is retrieved by the user. It was developed within the framework of HelpfulETH, a volunteering initiative of Swiss universities to support local hospitals during the COVID-19 pandemic. Starting from an urgent request of the University Hospital Zurich, the system has been developed within a time frame of five weeks through three consecutive sprints in close collaboration with medical professionals. After 18 days a first product increment (V 2.2) was delivered for daily use, confirming the general applicability and relevance of AHD for this specific context.

Within this study, the use of prototypes is analyzed with a visual tool, the Protomap [9]. It formalizes the product development process on the basis of employed prototypes (see Figure 2). The vertical axis depicts concepts that are developed in parallel, while the horizontal axis represents the iterations for each concept. Integrated physical increments are shown above the main horizontal axis. The resulting development history can be qualitatively analyzed regarding the use and adaptation of established prototyping strategies from $[7,8]$ due to the employed manufacturing technology. The identified practices are evaluated regarding their potential to increase development speed and reduce iteration run times.

\section{Results}

As depicted in Figure 2, various strategies from [7, 8] were applied to accelerate the embodiment of physical increments. In the following, they are illustrated with concrete examples.

\subsection{Iterative refinement}

As seen in the Protomap, multiple iterations were required for the development of the single product functions. These Design-Build-Test loops were mostly related to the technical refinement of the AM designs, i.e. iterating design parameters such as clearances by trial and error until a respective feature is sufficiently working. This is a common challenge in Design for AM (DfAM), as design knowledge is still largely dependent on personal expertise and experience of designers [10]. This need for functional refinement loops hinders the validation of the overall product's desirability, as for that typically all product functions must be realized within an integrated increment so that users can interact with it. Within this study, certain design features were deliberately kept simple in early iterations so that a tangible increment could be provided after every iteration. Only after user validation, the complexity of the feature was increased. One example for that is the blade mount (see Figure 3): In early iterations, a simple form fit design with a conventional razor blade was employed so that the overall product system could be validated. After doing so, a more sophisticated concept (press fit with surgical blade) was introduced. The design freedom of AM allowed for a flexible division and exchange of the increasingly more complex functional building blocks.

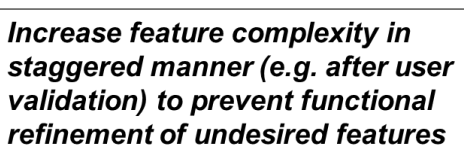

Increase feature complexity in refinement of undesired features

Figure 3: Feature complexity was deliberately kept low in early iterations 
Furthermore, functional refinement loops were avoided for certain design concepts with the help of a feature database for AM [11]. By reusing prior validated building blocks from the tool, the amount of iterations was reduced (see Iterative Refinement (2) in Figure 2) for features that were stored in the repository (e.g. snap fit). With an average lead time of 5 days for SLS parts within this project, avoiding additional iterations had an immediate impact on the acceleration of increment embodiment.

Trial and error

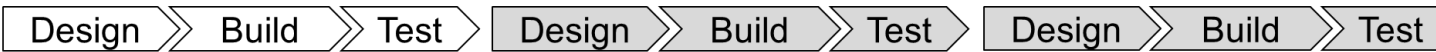

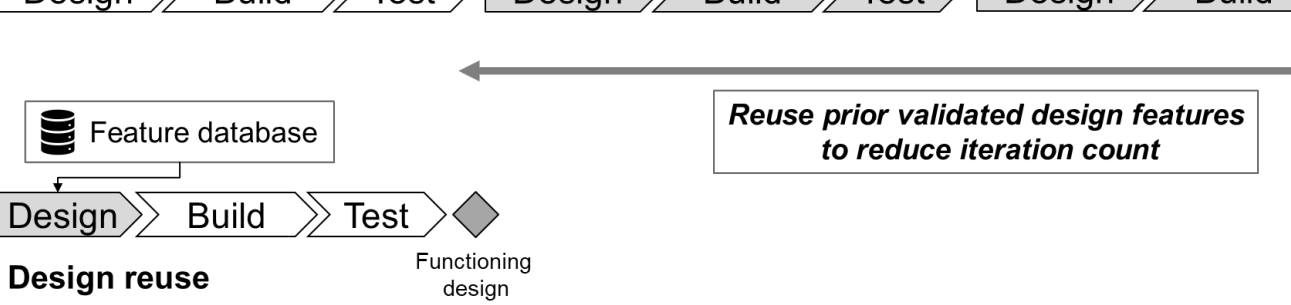

Figure 4: Schematic comparison of the applied design reuse approach vs. ordinary trial and error

\subsection{Parallel development of subsystems}

Within the case study, parallelization enabled to explore multiple, different design concepts for the same function. This was done either within the same iteration (see Parallel Prototyping (1) in Figure 2) or with alternating loops (2). For both cases, testing of physical prototypes ultimately determined which design concept would be continued, reaffirming the need for tangible product increments for early and frequent validation. A key challenge was to ensure compatibility when merging the multiple, in parallel developed AM concepts. To do so, comparable conditions at interfaces needed to be considered in prototyping design. This includes for instance similar building direction or cross sections for comparable thermal influx [12] at the merging interfaces (see Figure 5).

Master branch
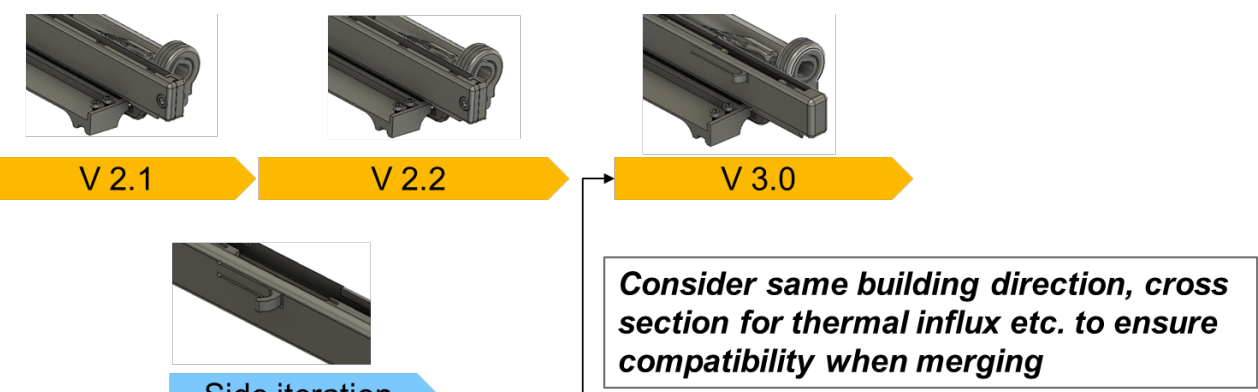

Side branch

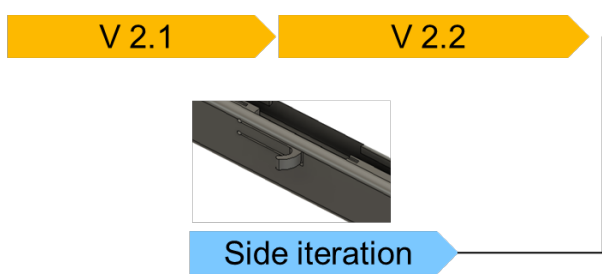
compatibility when merging

Figure 5: Parallel development of the blade shuttle guide with compatible merging interface

Furthermore, the subsystems were isolated in such a way that the variation of design parameters is limited to the smallest component. As seen in Figure 6, different design configurations of the sliding mechanism were incorporated within the small shuttle instead of the much bigger rail cover, minimizing manufacturing time and cost. 


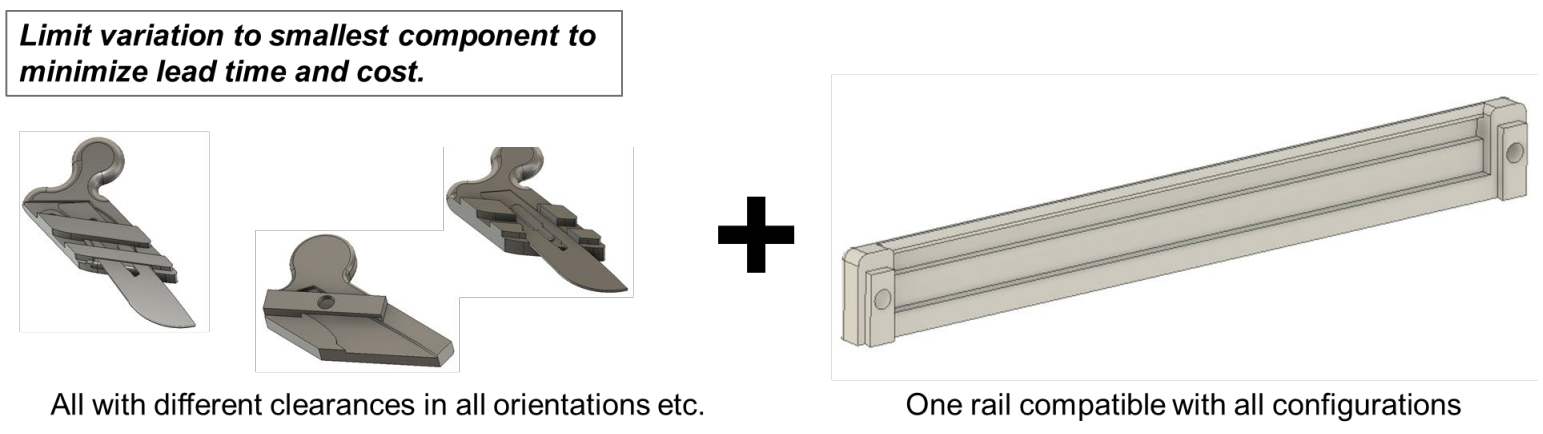

Figure 6: For parallel development, the variation of design parameters was limited to the smallest component.

\subsection{Change of prototyping media}

Prototyping media was systematically adjusted to minimize lead time and cost. In early iterations (V 1.0 - V 2.1), Fused Deposition Modeling (FDM) was used in combination with virtual prototypes (CAD animations) to quickly explore user needs. As uncertainty decreased, the prototyping objective moved towards the refinement of the functional part design and therefore to Selective Laser Sintering (SLS), as its part properties (i.e. material strength, resolution) were required for this application. For selected features, FDM parts were physically scaled to ensure similitude of part properties in relation to SLS (e.g. layer thickness, resolution). By doing so, certain concepts (such as the hinge mechanism in Figure 7) could already be tested with FDM parts and transferred to SLS, again enabling a faster build phase due to shorter lead times (in average 2 days for FDM compared to 5 days for SLS).

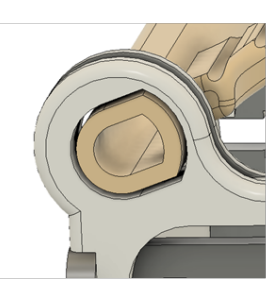

Example: Hinge

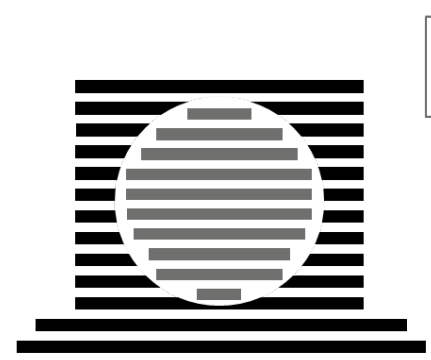

FDM part (Increased size)

\section{Scale prototype for similitude of certain part properties (e.g. layer thickness, resolution)}

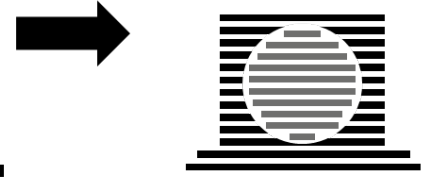

SLS part

(original size)

Figure 7: Scaled prototypes were used to ensure similitude of part properties during feature testing

\subsection{Scheduling of build phases}

In addition, the timing of manufacturing phases had a major impact on the prevention of delays. By aligning the build phases of the project to the production cadences of the AM supplier, lead time was optimally utilized. This was done for instance through selecting overnight or over the weekend manufacturing slots. As a consequence, the design and test phases of the iteration needed to be scheduled around the given manufacturing rhythm to prevent unnecessary downtimes.

\section{Discussion}

Overall, the application of the presented practices led to a vastly accelerated pace of increment embodiment. As a reference, the iteration length within this case amounted in average 6 days, while the first viable product increment was delivered to users after less than 18 days. By enabling early and frequent validations with tangible increments, the presented 
practices helped to address the CoP and therefore facilitated the effective application of AHD within this project.

The case study pointed out the importance of physical increments for AHD in general. They serve as an effective mean to reduce uncertainty while acting as a rapidly available, productive output for users. Especially the latter factor proved to be very important for the special context of this development project, as there was an urgent need for a technical solution from the very beginning. The various techniques identified within this study (summarized in Table 1) highlight that the development approach needs to be adapted in detail along the whole process chain. This finding reaffirms the matter that AHD encompasses much more than solely working in Sprints [13]. As seen in this study, established techniques from conventional prototyping strategies act as a good starting point for such an approach, which needs to be tailored for the specific context (product, technology, need).

Table 1: Prototyping strategies from [8] and their AM specific adaptation identified within this study

\begin{tabular}{ll}
\hline Prototyping strategy & AM specific adaptation \\
\hline Iterative refinement & $\begin{array}{l}\text { Increase feature complexity in a staggered manner (e.g. after user } \\
\text { validation) to prevent functional refinement of undesired features } \\
\text { Reuse prior validated building blocks to reduce iteration count }\end{array}$ \\
$\begin{array}{l}\text { Parallel prototyping of } \\
\text { subsystems }\end{array}$ & $\begin{array}{l}\text { Consider similar part properties at merging interface (e.g. building direction, } \\
\text { cross section for similar thermal influx) } \\
\text { Limit variation to smallest component to minimize lead time and cost } \\
\text { Media change }\end{array}$ \\
$\begin{array}{l}\text { Shift from quick AM process (e.g. FDM) for rapid exploration towards } \\
\text { functionally superior process for final design (if applicable) } \\
\text { Increase scale for similitude of part properties (e.g. layer thickness, } \\
\text { resolution) } \\
\text { Pcheduling of }\end{array}$ & $\begin{array}{l}\text { Plan design phases around the production cadences of the AM supplier to } \\
\text { optimally utilize lead time }\end{array}$ \\
\hline
\end{tabular}

\section{Conclusion and Outlook}

To sum up, the embodiment of physical increments within an AHD project can be facilitated through the use of technology specific prototyping strategies. Within this study, concrete practices within the area of iterative refinement, parallel development of subsystems, prototyping media change and scheduling of production phases have been found. They enable to accelerate, parallelize or prevent time-consuming and costly iterations and therefore address the CoP one typically faces in AHD. Compared to existing studies in the field of AHD with AM [1], the study provides concrete guidance on the overall product system level and not only on the level of single components.

In the future, the applicability of the described practices should be examined within an industrial setting. Especially the implications of AHD adoption on a firm's process chain with its separated organizational functions (Research \& Development $\rightarrow$ Operations $\rightarrow$ Marketing $\&$ Sales) would be of high interest. Furthermore, the transferability of the presented practices on other manufacturing processes apart from AM could be investigated aswell.

\section{References}

[1] Montero, Joaquin et. al.: Enhancing the Additive Manufacturing process for spare parts by applying Agile Hardware Development principles. In: ICMIMT 2019: IEEE, 2019, S. 109-116

[2] Solinski, Adam; Petersen, Kai: Prioritizing agile benefits and limitations in relation to practice usage. In: Software Quality Journal 24 (2016), Nr. 2, S. 447-482

[3] Schmidt, Tobias Sebastian; Paetzold, Kristin: Agilität als Alternative zu traditionellen Standards in der Entwicklung physischer Produkte: Chancen und Herausforderungen. In: DfX Symposium, 2016, S. 255-267 
[4] Riesener, Michael et. al.: Prototype Design in Agile Product Development Processes for Technical Systems. In: Proceedings of the ASME 2019 IDETC: ASME, 2019.

[5] Tuck, Christopher et. al.: Rapid manufacturing facilitated customization. In: International Journal of Computer Integrated Manufacturing 21 (2008), Nr. 3, S. 245-258

[6] Goh, Guo Dong et. al.: Additive manufacturing in unmanned aerial vehicles (UAVs): Challenges and potential. In: Aerospace Science and Technology 63 (2017), S. 140-151

[7] Christie, Edward James et. al.: Prototyping Strategies: Literature Review and Identification of Critical Variables. American Society for Engineering Education Conference, 2012.

[8] Camburn, Bradley et. al.: Design prototyping methods: state of the art in strategies, techniques, and guidelines. In: Design Science 3 (2017), S. 35

[9] Hansen, Camilla Arndt; Özkil, Ali Gürcan: From Idea to Production: A Retrospective and Longitudinal Case Study of Prototypes and Prototyping Strategies. In: Journal of Mechanical Design 142 (2020), Nr. 3, S. 117

[10] Kumke, Martin; Watschke, Hagen; Vietor, Thomas: A new methodological framework for design for additive manufacturing. In: Virtual and Physical Prototyping 11 (2016), Nr. 1, S. 3-19

[11] Omidvarkarjan, Daniel; Cipriano, Daniele; Rosenbauer, Ralph; Biedermann, Manuel; Meboldt, Mirko: Implementation of a design support tool for additive manufacturing using a feature database: an industrial case study. In: Progress in Additive Manufacturing 5 (2020), Nr. 1, S. 67-73

[12] Adam, Guido: Systematische Erarbeitung von Konstruktionsregeln für die additiven Fertigungsverfahren Lasersintern, Laserschmelzen und Fused Depostion Modeling. Paderborn, Universität Paderborn. Dissertation. 2015

[13] Omidvarkarjan, Daniel; Conrad, Jonas; Herbst, Constantin; Klahn, Christoph; Meboldt, Mirko: Bender - An Educational Game for Teaching Agile Hardware Development. In: Procedia Manufacturing 45 (2020), S. 313-318 